General Mathematics Vol. 27, No. 1 (2019), 57-69

DOI:10.2478/gm-2019-0006

\title{
sciendo
}

\section{Consensus of classical and fractional inequalities having congruity on time scale calculus ${ }^{1}$}

\author{
Muhammad Jibril Shahab Sahir
}

\begin{abstract}
In this paper, we find accordance of some classical inequalities and fractional dynamic inequalities. We find inequalities such as Radon's inequality, Bergström's inequality, Rogers-Hölder's inequality, Cauchy-Schwarz's inequality, the weighted power mean inequality and Schlömilch's inequality in generalized and extended form by using the Riemann-Liouville fractional integrals on time scales.
\end{abstract}

2010 Mathematics Subject Classification: 26D15, 26D20, 26A33, 39A12. Key words and phrases: Radon's inequality, Time scales, Riemann-Liouville fractional integrals.

\section{Introduction}

The inequality from (1) is called Bergström's inequality in literature as given in $[7,8,9,19]$.

Theorem 1 If $n \in \mathbb{N}, x_{k} \in \mathbb{R}$ and $y_{k}>0, k \in\{1,2, \ldots, n\}$, then

$$
\frac{\left(\sum_{k=1}^{n} x_{k}\right)^{2}}{\sum_{k=1}^{n} y_{k}} \leq \sum_{k=1}^{n} \frac{x_{k}^{2}}{y_{k}}
$$

with equality if and only if $\frac{x_{1}}{y_{1}}=\frac{x_{2}}{y_{2}}=\ldots=\frac{x_{n}}{y_{n}}$.

The upcoming result is called Radon's inequality as given in [20].

\footnotetext{
${ }^{1}$ Received 9 October, 2018

Accepted for publication (in revised form) 9 May, 2019
} 
Theorem 2 If $n \in \mathbb{N}, x_{k} \geq 0, y_{k}>0, k \in\{1,2, \ldots, n\}$ and $\beta \geq 0$, then

$$
\frac{\left(\sum_{k=1}^{n} x_{k}\right)^{\beta+1}}{\left(\sum_{k=1}^{n} y_{k}\right)^{\beta}} \leq \sum_{k=1}^{n} \frac{x_{k}^{\beta+1}}{y_{k}^{\beta}} .
$$

Inequality (2) is widely studied by many authors because it is used in many applications.

The following inequality is generalized Radon's inequality as given in [15].

Theorem 3 If $n \in \mathbb{N}, x_{k} \geq 0, y_{k}>0, k \in\{1,2, \ldots, n\}, \beta \geq 0$ and $\gamma \geq 1$, then

$$
\frac{\left(\sum_{k=1}^{n} x_{k} y_{k}^{\gamma-1}\right)^{\beta+\gamma}}{\left(\sum_{k=1}^{n} y_{k}^{\gamma}\right)^{\beta+\gamma-1}} \leq \sum_{k=1}^{n} \frac{x_{k}^{\beta+\gamma}}{y_{k}^{\beta}},
$$

with equality if and only if $\frac{x_{1}}{y_{1}}=\frac{x_{2}}{y_{2}}=\ldots=\frac{x_{n}}{y_{n}}$.

We will prove these results on time scales. The calculus of time scales was initiated by Stefan Hilger as given in [17]. A time scale is an arbitrary nonempty closed subset of the real numbers. The theory of time scales is applied to unify discrete and continuous analysis and to combine them in one comprehensive form. In time scale calculus, results are unified and extended. This hybrid theory is also widely applied on dynamic inequalities. Basic work on dynamic inequalities is done by Ravi Agarwal, George Anastassiou, Martin Bohner, Allan Peterson, Donal O'Regan, Samir Saker and many other authors.

In this paper, it is assumed that all considerable integrals exist and are finite and $\mathbb{T}$ is a time scale, $a, b \in \mathbb{T}$ with $a<b$ and an interval $[a, b]_{\mathbb{T}}$ means the intersection of a real interval with the given time scale.

\section{Preliminaries}

We need here basic concepts of delta calculus. The results of delta calculus are adapted from monographs $[10,11]$.

For $t \in \mathbb{T}$, the forward jump operator $\sigma: \mathbb{T} \rightarrow \mathbb{T}$ is defined by

$$
\sigma(t):=\inf \{s \in \mathbb{T}: s>t\} .
$$

The mapping $\mu: \mathbb{T} \rightarrow \mathbb{R}_{0}^{+}=[0,+\infty)$ such that $\mu(t):=\sigma(t)-t$ is called the forward graininess function. The backward jump operator $\rho: \mathbb{T} \rightarrow \mathbb{T}$ is defined by

$$
\rho(t):=\sup \{s \in \mathbb{T}: s<t\} .
$$


The mapping $\nu: \mathbb{T} \rightarrow \mathbb{R}_{0}^{+}=[0,+\infty)$ such that $\nu(t):=t-\rho(t)$ is called the backward graininess function. If $\sigma(t)>t$, we say that $t$ is right-scattered, while if $\rho(t)<t$, we say that $t$ is left-scattered. Also, if $t<\sup \mathbb{T}$ and $\sigma(t)=t$, then $t$ is called right-dense, and if $t>\inf \mathbb{T}$ and $\rho(t)=t$, then $t$ is called left-dense. If $\mathbb{T}$ has a left-scattered maximum $M$, then $\mathbb{T}^{k}=\mathbb{T}-\{M\}$, otherwise $\mathbb{T}^{k}=\mathbb{T}$.

For a function $f: \mathbb{T} \rightarrow \mathbb{R}$, the delta derivative $f^{\Delta}$ is defined as follows:

Let $t \in \mathbb{T}^{k}$, if there exists $f^{\Delta}(t) \in \mathbb{R}$ such that for all $\epsilon>0$, there exists a neighborhood $U$ of $t$, such that

$$
\left|f(\sigma(t))-f(s)-f^{\Delta}(t)(\sigma(t)-s)\right| \leq \epsilon|\sigma(t)-s|,
$$

for all $s \in U$, then $f$ is said to be delta differentiable at $t$, and $f^{\Delta}(t)$ is called the delta derivative of $f$ at $t$.

A function $f: \mathbb{T} \rightarrow \mathbb{R}$ is said to be right-dense continuous (rd-continuous), if it is continuous at each right-dense point and there exists a finite left limit at every left-dense point. The set of all rd-continuous functions is denoted by $C_{r d}(\mathbb{T}, \mathbb{R})$.

The next definition is given in $[10,11]$.

Definition 1 A function $F: \mathbb{T} \rightarrow \mathbb{R}$ is called a delta antiderivative of $f: \mathbb{T} \rightarrow \mathbb{R}$, provided that $F^{\Delta}(t)=f(t)$ holds for all $t \in \mathbb{T}^{k}$. Then the delta integral of $f$ is defined by

$$
\int_{a}^{b} f(t) \Delta t=F(b)-F(a) .
$$

The following results of nabla calculus are taken from $[6,10,11]$.

If $\mathbb{T}$ has a right-scattered minimum $m$, then $\mathbb{T}_{k}=\mathbb{T}-\{m\}$, otherwise $\mathbb{T}_{k}=\mathbb{T}$. A function $f: \mathbb{T}_{k} \rightarrow \mathbb{R}$ is called nabla differentiable at $t \in \mathbb{T}_{k}$, with nabla derivative $f^{\nabla}(t)$, if there exists $f^{\nabla}(t) \in \mathbb{R}$ such that for any $\epsilon>0$, there exists a neighborhood $V$ of $t$, such that

$$
\left|f(\rho(t))-f(s)-f^{\nabla}(t)(\rho(t)-s)\right| \leq \epsilon|\rho(t)-s|,
$$

for all $s \in V$.

A function $f: \mathbb{T} \rightarrow \mathbb{R}$ is said to be left-dense continuous (ld-continuous), provided it is continuous at all left-dense points in $\mathbb{T}$ and its right-sided limits exist (finite) at all right-dense points in $\mathbb{T}$. The set of all ld-continuous functions is denoted by $C_{l d}(\mathbb{T}, \mathbb{R})$.

The next definition is given in $[6,10,11]$.

Definition 2 A function $G: \mathbb{T} \rightarrow \mathbb{R}$ is called a nabla antiderivative of $g: \mathbb{T} \rightarrow \mathbb{R}$, provided that $G^{\nabla}(t)=g(t)$ holds for all $t \in \mathbb{T}_{k}$. Then the nabla integral of $g$ is defined by

$$
\int_{a}^{b} g(t) \nabla t=G(b)-G(a) .
$$


We need the following results.

Definition 3 [14]. A function $f: \mathbb{T} \rightarrow \mathbb{R}$ is called convex on $I_{\mathbb{T}}=I \cap \mathbb{T}$, where $I$ is an interval of $\mathbb{R}$ (open or closed), if

$$
f(\lambda t+(1-\lambda) s) \leq \lambda f(t)+(1-\lambda) f(s),
$$

for all $t, s \in I_{\mathbb{T}}$ and all $\lambda \in[0,1]$ such that $\lambda t+(1-\lambda) s \in I_{\mathbb{T}}$.

The function $f$ is strictly convex on $I_{\mathbb{T}}$ if the inequality (4) is strict for distinct $t, s \in I_{\mathbb{T}}$ and $\lambda \in(0,1)$.

The function $f$ is concave (respectively, strictly concave) on $I_{\mathbb{T}}$, if - $f$ is convex (respectively, strictly convex).

Theorem 4 [2]. Let $a, b \in \mathbb{T}$ and $c, d \in \mathbb{R}$. Suppose that $g \in C_{r d}\left([a, b]_{\mathbb{T}},(c, d)\right)$ and $w \in C_{r d}\left([a, b]_{\mathbb{T}}, \mathbb{R}\right)$ with $\int_{a}^{b}|w(s)| \Delta s>0$. If $\Psi \in C((c, d), \mathbb{R})$ is convex, then the generalized Jensen's inequality is

$$
\Psi\left(\frac{\int_{a}^{b}|w(s)| g(s) \Delta s}{\int_{a}^{b}|w(s)| \Delta s}\right) \leq \frac{\int_{a}^{b}|w(s)| \Psi(g(s)) \Delta s}{\int_{a}^{b}|w(s)| \Delta s} .
$$

If $\Psi$ is strictly convex, then the inequality sign " $\leq$ " in the above inequality should be replaced by " $<$ ".

We need the following definitions of $\Delta$-Riemann-Liouville type fractional integral and $\nabla$-Riemann-Liouville type fractional integral.

The following definition is taken from $[3,5]$.

Definition 4 For $\alpha \geq 1$, the time scale $\Delta$-Riemann-Liouville type fractional integral is defined by

$$
I_{a}^{\alpha} f(t)=\int_{a}^{t} h_{\alpha-1}(t, \sigma(\tau)) f(\tau) \Delta \tau,
$$

which is an integral on $[a, t)_{\mathbb{T}}$, as given in [12] and $h_{\alpha}: \mathbb{T} \times \mathbb{T} \rightarrow \mathbb{R}, \alpha \geq 0$ are the coordinate wise rd-continuous functions, such that $h_{0}(t, s)=1$,

$$
h_{\alpha+1}(t, s)=\int_{s}^{t} h_{\alpha}(\tau, s) \Delta \tau, \forall s, t \in \mathbb{T} .
$$

Notice

$$
I_{a}^{1} f(t)=\int_{a}^{t} f(\tau) \Delta \tau,
$$

which is absolutely continuous in $t \in[a, b]_{\mathbb{T}}$, as in [12]. 
The following definition is taken from $[4,5]$.

Definition 5 For $\alpha \geq 1$, the time scale $\nabla$-Riemann-Liouville type fractional integral is defined by

$$
J_{a}^{\alpha} f(t)=\int_{a}^{t} \hat{h}_{\alpha-1}(t, \rho(\tau)) f(\tau) \nabla \tau
$$

which is an integral on $(a, t]_{\mathbb{T}}$, as given in [12] and $\hat{h}_{\alpha}: \mathbb{T} \times \mathbb{T} \rightarrow \mathbb{R}, \alpha \geq 0$ are the coordinate wise ld-continuous functions, such that $\hat{h}_{0}(t, s)=1$,

$$
\hat{h}_{\alpha+1}(t, s)=\int_{s}^{t} \hat{h}_{\alpha}(\tau, s) \nabla \tau, \forall s, t \in \mathbb{T} .
$$

Notice

$$
J_{a}^{1} f(t)=\int_{a}^{t} f(\tau) \nabla \tau
$$

which is absolutely continuous in $t \in[a, b]_{\mathbb{T}}$, as in [12].

\section{Main results}

In order to present our main results, first we prove an extension of Radon's inequality by using the time scale Riemann-Liouville type fractional integral.

Theorem 5 Let $w, f, g \in C_{r d}\left([a, b]_{\mathbb{T}}, \mathbb{R}\right)$ and neither $w \equiv 0$ nor $g \equiv 0$.

(i) If $\beta \geq 0$ and $\gamma \geq 1$, then for $\alpha \geq 1$

$$
\frac{\left\{I_{a}^{\alpha}\left(|w(x)||f(x)||g(x)|^{\gamma-1}\right)\right\}^{\beta+\gamma}}{\left\{I_{a}^{\alpha}\left(|w(x) \| g(x)|^{\gamma}\right)\right\}^{\beta+\gamma-1}} \leq I_{a}^{\alpha}\left\{\frac{|w(x)||f(x)|^{\beta+\gamma}}{|g(x)|^{\beta}}\right\} .
$$

(ii) If $0<\beta+\gamma<1$, then for $\alpha \geq 1$

$$
\frac{\left\{I_{a}^{\alpha}\left(|w(x)||f(x) \| g(x)|^{\gamma-1}\right)\right\}^{\beta+\gamma}}{\left\{I_{a}^{\alpha}\left(|w(x) \| g(x)|^{\gamma}\right)\right\}^{\beta+\gamma-1}} \geq I_{a}^{\alpha}\left\{\frac{|w(x)||f(x)|^{\beta+\gamma}}{|g(x)|^{\beta}}\right\} .
$$

Equality holds in both (10) and (11) if and only if $f=c g$, where $c$ is a real constant. 
Proof. Case (i). Choosing $\Psi(x)=x^{\beta+\gamma}$, which for $\beta \geq 0$ and $\gamma \geq 1$ is a convex function on $[0,+\infty)$. Replacing $g(y)$ by $\left|\frac{f(y)}{g(y)}\right|$ and $|w(y)|$ by $h_{\alpha-1}(x, \sigma(y))|w(y)||g(y)|^{\gamma}$ for $h_{\alpha-1}(x, \sigma(y))>0$ in Theorem 4, we get

$$
\begin{gathered}
\left(\int_{a}^{x} \frac{h_{\alpha-1}(x, \sigma(y))|w(y)||g(y)|^{\gamma}}{\int_{a}^{x} h_{\alpha-1}(x, \sigma(y))|w(y)||g(y)|^{\gamma \Delta y}}\left|\frac{f(y)}{g(y)}\right| \Delta y\right)^{\beta+\gamma} \\
\leq \frac{\int_{a}^{x} h_{\alpha-1}(x, \sigma(y))|w(y)||g(y)|^{\gamma}\left|\frac{f(y)}{g(y)}\right|^{\beta+\gamma} \Delta y}{\int_{a}^{x} h_{\alpha-1}(x, \sigma(y))|w(y)||g(y)|^{\gamma} \Delta y}
\end{gathered}
$$

$\forall x \in[a, b]_{\mathbb{T}}$. Then

$$
\frac{\left\{I_{a}^{\alpha}\left(|w(x)\|f(x)\| g(x)|^{\gamma-1}\right)\right\}^{\beta+\gamma}}{\left\{I_{a}^{\alpha}\left(|w(x) \| g(x)|^{\gamma}\right)\right\}^{\beta+\gamma}} \leq \frac{I_{a}^{\alpha}\left(\frac{|w(x)||f(x)|^{\beta+\gamma}}{|g(x)|^{\beta}}\right)}{I_{a}^{\alpha}\left(|w(x)||g(x)|^{\gamma}\right)} .
$$

Multiplying both sides of the last inequality by $I_{a}^{\alpha}\left(|w(x)||g(x)|^{\gamma}\right)$, we get the desired inequality given in (10).

Case (ii). Similarly, the inequality given in (10) is reversed for $0<\beta+\gamma<1$.

It is clear that the equality holds in both (10) and (11) if and only if $f=c g$, where $c$ is a real constant.

Thus, the proof of Theorem 5 is completed.

The following result is a nabla version of Radon's inequality by using the time scale Riemann-Liouville type fractional integral.

Theorem 6 Let $w, f, g \in C_{l d}\left([a, b]_{\mathbb{T}}, \mathbb{R}\right)$ and neither $w \equiv 0$ nor $g \equiv 0$.

(i) If $\beta \geq 0$ and $\gamma \geq 1$, then for $\alpha \geq 1$

$$
\frac{\left\{J_{a}^{\alpha}\left(|w(x)||f(x)||g(x)|^{\gamma-1}\right)\right\}^{\beta+\gamma}}{\left\{J_{a}^{\alpha}\left(|w(x) \| g(x)|^{\gamma}\right)\right\}^{\beta+\gamma-1}} \leq J_{a}^{\alpha}\left\{\frac{|w(x)||f(x)|^{\beta+\gamma}}{|g(x)|^{\beta}}\right\} .
$$

(ii) If $0<\beta+\gamma<1$, then for $\alpha \geq 1$

$$
\frac{\left\{J_{a}^{\alpha}\left(|w(x)||f(x)||g(x)|^{\gamma-1}\right)\right\}^{\beta+\gamma}}{\left\{J_{a}^{\alpha}\left(|w(x)||g(x)|^{\gamma}\right)\right\}^{\beta+\gamma-1}} \geq J_{a}^{\alpha}\left\{\frac{|w(x)||f(x)|^{\beta+\gamma}}{|g(x)|^{\beta}}\right\} .
$$

Equality holds in both (14) and (15) if and only if $f=c g$, where $c$ is a real constant.

Proof. Similar to the proof of Theorem 5.

Remark 1 If $\alpha=1$, then $I_{a}^{1} f(x)=\int_{a}^{x} f(y) \Delta y, \forall x \in[a, b]_{\mathbb{T}}$. Further we set $\mathbb{T}=\mathbb{Z}$, $a=1, x=b=n+1, w \equiv 1, f(k)=x_{k} \in \mathbb{R}$ and $g(k)=y_{k} \in(0,+\infty)$ for $k \in\{1,2, \ldots, n\}, n \in \mathbb{N}$. If $\beta=1$ and $\gamma=1$, then (10) reduces to (1). 
Remark 2 If $\alpha=1$, then $I_{a}^{1} f(x)=\int_{a}^{x} f(y) \Delta y, \forall x \in[a, b]_{\mathbb{T}}$. Further we set $\mathbb{T}=\mathbb{Z}$, $a=1, x=b=n+1, w \equiv 1, f(k)=x_{k} \in[0,+\infty)$ and $g(k)=y_{k} \in(0,+\infty)$ for $k \in\{1,2, \ldots, n\}, n \in \mathbb{N}$. If $\gamma=1$, then (10) reduces to (2) and we get reverse version of (2) for $-1<\beta<0$.

Remark 3 If $\alpha=1$, then $I_{a}^{1} f(x)=\int_{a}^{x} f(y) \Delta y, \forall x \in[a, b]_{\mathbb{T}}$. If $\mathbb{T}=\mathbb{Z}, a=1$, $x=b=n+1, w \equiv 1, f(k)=x_{k} \in[0,+\infty)$ and $g(k)=y_{k} \in(0,+\infty)$ for $k \in\{1,2, \ldots, n\}, n \in \mathbb{N}$, then discrete version of (10) reduces to (3) and we get reverse discrete version of (3) for $0<\beta+\gamma<1$.

Also if $\alpha=1$ and $\mathbb{T}=\mathbb{R}$, then continuous version of (10) takes the form

$$
\frac{\left(\int_{a}^{x}|w(y)||f(y)||g(y)|^{\gamma-1} d y\right)^{\beta+\gamma}}{\left(\int_{a}^{x}|w(y)||g(y)|^{\gamma} d y\right)^{\beta+\gamma-1}} \leq \int_{a}^{x} \frac{|w(y)||f(y)|^{\beta+\gamma}}{|g(y)|^{\beta}} d y .
$$

Next, we give the following extension of Rogers-Hölder's inequality by using the time scale Riemann-Liouville type fractional integral.

Corollary 1 Let $w, f, g \in C_{r d}\left([a, b]_{\mathbb{T}}, \mathbb{R}\right)$. If $\frac{1}{p}+\frac{1}{q}=1$ with $p>1$, then for $\alpha \geq 1$

$$
I_{a}^{\alpha}(|w(x)||f(x) g(x)|) \leq\left\{I_{a}^{\alpha}\left(|w(x)||f(x)|^{p}\right)\right\}^{\frac{1}{p}}\left\{I_{a}^{\alpha}\left(|w(x) \| g(x)|^{q}\right)\right\}^{\frac{1}{q}} .
$$

Proof. Inequality (17) is trivially true in the case when $w$ or $f$ or $g$ is identically zero. If $\beta>0, \gamma=1$ and $\beta+1=p>1$, then (10) takes the form

$$
\frac{\left\{I_{a}^{\alpha}(|w(x)||f(x)|)\right\}^{p}}{\left\{I_{a}^{\alpha}(|w(x)||g(x)|)\right\}^{p-1}} \leq I_{a}^{\alpha}\left\{\frac{|w(x)||f(x)|^{p}}{|g(x)|^{p-1}}\right\}
$$

Replacing $|w(x)|$ by $|w(x)||g(x)|^{p-1}$ in (18), we obtain

$$
\frac{\left\{I_{a}^{\alpha}\left(|w(x)\|f(x)\| g(x)|^{p-1}\right)\right\}^{p}}{\left\{I_{a}^{\alpha}\left(|w(x) \| g(x)|^{p}\right)\right\}^{p-1}} \leq I_{a}^{\alpha}\left(|w(x) \| f(x)|^{p}\right) .
$$

Taking power $\frac{1}{p}>0$ on both sides of (19), we get

$$
I_{a}^{\alpha}\left(|w(x)||f(x) \| g(x)|^{p-1}\right) \leq\left\{I_{a}^{\alpha}\left(|w(x) \| f(x)|^{p}\right)\right\}^{\frac{1}{p}}\left\{I_{a}^{\alpha}\left(|w(x) \| g(x)|^{p}\right)\right\}^{1-\frac{1}{p}} .
$$

Replacing $|g(x)|$ by $|g(x)|^{\frac{q}{p}}$ and using the fact that $\frac{1}{p}+\frac{1}{q}=1$, we get our desired result.

The following result is a delta version of Cauchy-Schwarz's inequality by using the time scale Riemann-Liouville type fractional integral.

Corollary 2 Let $w, f, g \in C_{r d}\left([a, b]_{\mathbb{T}}, \mathbb{R}\right)$. Then for $\alpha \geq 1$

$$
I_{a}^{\alpha}(|w(x) \| f(x) g(x)|) \leq\left\{I_{a}^{\alpha}\left(|w(x)||f(x)|^{2}\right)\right\}^{\frac{1}{2}}\left\{I_{a}^{\alpha}\left(|w(x)||g(x)|^{2}\right)\right\}^{\frac{1}{2}} .
$$


Proof. Putting $p=q=2$ in (17), we get (21).

Remark 4 If $\mathbb{T}=\mathbb{R}$ and $w \equiv 1$, then continuous version of (17) takes the form

$$
I_{a}^{\alpha}|f(x) g(x)| \leq\left(I_{a}^{\alpha}|f(x)|^{p}\right)^{\frac{1}{p}}\left(I_{a}^{\alpha}|g(x)|^{q}\right)^{\frac{1}{q}} .
$$

For $a=0$, the inequality (22) is similar to an inequality of [13, Theorem 3].

Now we present a nabla version of Rogers-Hölder's inequality by using the time scale Riemann-Liouville type fractional integral.

Corollary 3 Let $w, f, g \in C_{l d}\left([a, b]_{\mathbb{T}}, \mathbb{R}\right)$. If $\frac{1}{p}+\frac{1}{q}=1$ with $p>1$, then for $\alpha \geq 1$

$$
J_{a}^{\alpha}(|w(x) \| f(x) g(x)|) \leq\left\{J_{a}^{\alpha}\left(|w(x) \| f(x)|^{p}\right)\right\}^{\frac{1}{p}}\left\{J_{a}^{\alpha}\left(|w(x) \| g(x)|^{q}\right)\right\}^{\frac{1}{q}} .
$$

Proof. Similar to the proof of Corollary 1.

The upcoming result is a nabla version of Cauchy-Schwarz's inequality by using the time scale Riemann-Liouville type fractional integral.

Corollary 4 Let $w, f, g \in C_{l d}\left([a, b]_{\mathbb{T}}, \mathbb{R}\right)$. Then for $\alpha \geq 1$

$$
J_{a}^{\alpha}(|w(x)||f(x) g(x)|) \leq\left\{J_{a}^{\alpha}\left(|w(x)||f(x)|^{2}\right)\right\}^{\frac{1}{2}}\left\{J_{a}^{\alpha}\left(|w(x)||g(x)|^{2}\right)\right\}^{\frac{1}{2}} .
$$

Proof. Putting $p=q=2$ in (23), we get (24).

Corollary 5 Let $w, f, g, h \in C_{r d}\left([a, b]_{\mathbb{T}}, \mathbb{R}-\{0\}\right)$ satisfy $f g h=M$, where $M$ is a positive real number and let $\frac{1}{p}+\frac{1}{q}+\frac{1}{r}=0$ with $p, q, r \in \mathbb{R}-\{0\}$.

(i) If all but one of $p, q, r$ are positive, then for $\alpha \geq 1$

$$
\left\{I_{a}^{\alpha}\left(|w(x)||f(x)|^{p}\right)\right\}^{\frac{1}{p}}\left\{I_{a}^{\alpha}\left(|w(x)||g(x)|^{q}\right)\right\}^{\frac{1}{q}}\left\{I_{a}^{\alpha}\left(|w(x) \| h(x)|^{r}\right)\right\}^{\frac{1}{r}} \geq M .
$$

(ii) If all but one of $p, q, r$ are negative, then for $\alpha \geq 1$

$$
\left\{I_{a}^{\alpha}\left(|w(x) \| f(x)|^{p}\right)\right\}^{\frac{1}{p}}\left\{I_{a}^{\alpha}\left(|w(x)||g(x)|^{q}\right)\right\}^{\frac{1}{q}}\left\{I_{a}^{\alpha}\left(|w(x) \| h(x)|^{r}\right)\right\}^{\frac{1}{r}} \leq M .
$$

Proof. Case (i). Let $p>0, q>0$ but $r<0$. From $\frac{1}{p}+\frac{1}{q}+\frac{1}{r}=0$, we find that $\frac{1}{\left(-\frac{p}{r}\right)}+\frac{1}{\left(-\frac{q}{r}\right)}=1$, where $-\frac{p}{r}>1$ and $-\frac{q}{r}>1$.

The rest follows as in the proof of Corollary 1.

Similarly, we can prove the Case (ii).

Remark 5 If $\alpha=1, \mathbb{T}=\mathbb{Z}, a=1, x=b=n+1, w \equiv 1, f(k)=x_{k}, g(k)=y_{k}$, $h(k)=z_{k}$ for $k \in\{1,2, \ldots, n\}, n \in \mathbb{N}$, where $x_{k}, y_{k}, z_{k}$ are the sets of positive values and $M=1$, then discrete version of (25) takes the form

$$
\left(\sum_{k=1}^{n} x_{k}^{p}\right)^{\frac{1}{p}}\left(\sum_{k=1}^{n} y_{k}^{q}\right)^{\frac{1}{q}}\left(\sum_{k=1}^{n} z_{k}^{r}\right)^{\frac{1}{r}} \geq 1
$$


and discrete version of (26) becomes

$$
\left(\sum_{k=1}^{n} x_{k}^{p}\right)^{\frac{1}{p}}\left(\sum_{k=1}^{n} y_{k}^{q}\right)^{\frac{1}{q}}\left(\sum_{k=1}^{n} z_{k}^{r}\right)^{\frac{1}{r}} \leq 1 .
$$

Inequalities (27) and (28) are given in [1, page 147].

Remark 6 If $M=1$, then inequality (25) reduces to inequality (3.1) of [22].

Corollary 6 Let $w, f, g, h \in C_{l d}\left([a, b]_{\mathbb{T}}, \mathbb{R}-\{0\}\right)$ satisfy $f g h=M$, where $M$ is a positive real number and let $\frac{1}{p}+\frac{1}{q}+\frac{1}{r}=0$ with $p, q, r \in \mathbb{R}-\{0\}$.

(i) If all but one of $p, q, r$ are positive, then for $\alpha \geq 1$

(29) $\left\{J_{a}^{\alpha}\left(|w(x) \| f(x)|^{p}\right)\right\}^{\frac{1}{p}}\left\{J_{a}^{\alpha}\left(|w(x) \| g(x)|^{q}\right)\right\}^{\frac{1}{q}}\left\{J_{a}^{\alpha}\left(|w(x)||h(x)|^{r}\right)\right\}^{\frac{1}{r}} \geq M$.

(ii) If all but one of $p, q, r$ are negative, then for $\alpha \geq 1$

(30) $\left\{J_{a}^{\alpha}\left(|w(x)||f(x)|^{p}\right)\right\}^{\frac{1}{p}}\left\{J_{a}^{\alpha}\left(|w(x) \| g(x)|^{q}\right)\right\}^{\frac{1}{q}}\left\{J_{a}^{\alpha}\left(|w(x)||h(x)|^{r}\right)\right\}^{\frac{1}{r}} \leq M$.

Proof. Similar to the proof of Corollary 5.

The upcoming result is a delta version of the weighted power mean inequality by using the time scale Riemann-Liouville type fractional integral.

Corollary 7 Let $w, f \in C_{r d}\left([a, b]_{\mathbb{T}}, \mathbb{R}\right)$ with $I_{a}^{\alpha}|w(x)|>0$. If $\eta_{2} \geq \eta_{1}>0$, then for $\alpha \geq 1$

$$
\left\{\frac{I_{a}^{\alpha}\left(|w(x)||f(x)|^{\eta_{1}}\right)}{I_{a}^{\alpha}|w(x)|}\right\}^{\frac{1}{\eta_{1}}} \leq\left\{\frac{I_{a}^{\alpha}\left(|w(x)||f(x)|^{\eta_{2}}\right)}{I_{a}^{\alpha}|w(x)|}\right\}^{\frac{1}{\eta_{2}}} .
$$

Proof. If $\beta \geq 0$ and $\gamma \geq 1$, then $\beta+\gamma=\frac{\eta_{2}}{\eta_{1}} \geq 1$ and if $g \equiv 1$, then (10) becomes

$$
\frac{\left\{I_{a}^{\alpha}(|w(x)||f(x)|)\right\}^{\frac{\eta_{2}}{\eta_{1}}}}{\left(I_{a}^{\alpha}|w(x)|\right)^{\frac{\eta_{2}}{\eta_{1}}-1}} \leq I_{a}^{\alpha}\left(|w(x)||f(x)|^{\frac{\eta_{2}}{\eta_{1}}}\right) .
$$

Dividing both sides of (32) by $I_{a}^{\alpha}|w(x)|$, replacing $|f(x)|$ by $|f(x)|^{\eta_{1}}$ and taking power $\frac{1}{\eta_{2}}>0$, we obtain the desired result.

Remark 7 If $\alpha=1, \mathbb{T}=\mathbb{Z}, a=1, x=b=n+1, w(k)$ is replaced by $p_{k} \in(0,+\infty)$ and $f(k)$ is replaced by $x_{k} \in[0,+\infty)$ for $k \in\{1,2, \ldots, n\}, n \in \mathbb{N}$, then (31) reduces to

$$
\left(\frac{p_{1} x_{1}^{\eta_{1}}+p_{2} x_{2}^{\eta_{1}}+\ldots+p_{n} x_{n}^{\eta_{1}}}{p_{1}+p_{2}+\ldots+p_{n}}\right)^{\frac{1}{\eta_{1}}} \leq\left(\frac{p_{1} x_{1}^{\eta_{2}}+p_{2} x_{2}^{\eta_{2}}+\ldots+p_{n} x_{n}^{\eta_{2}}}{p_{1}+p_{2}+\ldots+p_{n}}\right)^{\frac{1}{\eta_{2}}}
$$

which is called the weighted power mean inequality as given in [16]. 
Corollary 8 Let $w, f \in C_{l d}\left([a, b]_{\mathbb{T}}, \mathbb{R}\right)$ with $J_{a}^{\alpha}|w(x)|>0$. If $\eta_{2} \geq \eta_{1}>0$, then for $\alpha \geq 1$

$$
\left\{\frac{J_{a}^{\alpha}\left(|w(x)||f(x)|^{\eta_{1}}\right)}{J_{a}^{\alpha}|w(x)|}\right\}^{\frac{1}{\eta_{1}}} \leq\left\{\frac{J_{a}^{\alpha}\left(|w(x)||f(x)|^{\eta_{2}}\right)}{J_{a}^{\alpha}|w(x)|}\right\}^{\frac{1}{\eta_{2}}} .
$$

Proof. Similar to the proof of Corollary 7.

Example 1 If $\alpha=1, w \equiv 1$ and $\eta_{1}=1$, then (31) takes the form

$$
\left(\frac{1}{x-a}\right)^{\eta_{2}-1}\left(\int_{a}^{x}|f(y)| \Delta y\right)^{\eta_{2}} \leq \int_{a}^{x}|f(y)|^{\eta_{2}} \Delta y
$$

and (34) takes the form

$$
\left(\frac{1}{x-a}\right)^{\eta_{2}-1}\left(\int_{a}^{x}|f(y)| \nabla y\right)^{\eta_{2}} \leq \int_{a}^{x}|f(y)|^{\eta_{2}} \nabla y
$$

Corollary 9 Let $w, f \in C_{r d}\left([a, b]_{\mathbb{T}}, \mathbb{R}\right)$ with $I_{a}^{\alpha}|w(b)|=1$. If $\eta_{2} \geq \eta_{1}>0$, then for $\alpha \geq 1$

$$
\left\{I_{a}^{\alpha}\left(|w(b)||f(b)|^{\eta_{1}}\right)\right\}^{\frac{1}{\eta_{1}}} \leq\left\{I_{a}^{\alpha}\left(|w(b)||f(b)|^{\eta_{2}}\right)\right\}^{\frac{1}{\eta_{2}}} .
$$

Proof. Putting $x=b$ in (31), we get (37).

Inequality (37) is called Schlömilch's inequality by using the time scale RiemannLiouville type fractional integral. Its other versions are also given in $[16,18,21]$.

Remark 8 If $\alpha=1, \mathbb{T}=\mathbb{Z}, a=1, x=b=n+1, w \equiv \frac{1}{n}, f(k)=x_{k} \in(0,+\infty)$, $k \in\{1,2, \ldots, n\}, n \in \mathbb{N}$ and $\eta_{1}<\eta_{2}$, then (37) reduces to

$$
\left(\frac{1}{n} \sum_{k=1}^{n} x_{k}^{\eta_{1}}\right)^{\frac{1}{\eta_{1}}}<\left(\frac{1}{n} \sum_{k=1}^{n} x_{k}^{\eta_{2}}\right)^{\frac{1}{\eta_{2}}}
$$

unless the $x_{k}$ for $k \in \mathbb{N}$ are all equal.

The inequality from (38) is called Schlömilch's inequality in literature as given in $[16, p .26]$.

Corollary 10 Let $w, f \in C_{l d}\left([a, b]_{\mathbb{T}}, \mathbb{R}\right)$ with $J_{a}^{\alpha}|w(b)|=1$. If $\eta_{2} \geq \eta_{1}>0$, then for $\alpha \geq 1$

$$
\left\{J_{a}^{\alpha}\left(|w(b)||f(b)|^{\eta_{1}}\right)\right\}^{\frac{1}{\eta_{1}}} \leq\left\{J_{a}^{\alpha}\left(|w(b)||f(b)|^{\eta_{2}}\right)\right\}^{\frac{1}{\eta_{2}}} .
$$

Proof. Putting $x=b$ in (34), we get (39).

Corollary 11 Let $w, f, g \in C_{r d}\left([a, b]_{\mathbb{T}}, \mathbb{R}\right)$ with $I_{a}^{\alpha}|w(b)|=1$. If $p>1, q>1$ with $\frac{1}{p}+\frac{1}{q}<1$, then for $\alpha \geq 1$

$$
I_{a}^{\alpha}(|w(b)||f(b) g(b)|) \leq\left\{I_{a}^{\alpha}\left(|w(b) \| f(b)|^{p}\right)\right\}^{\frac{1}{p}}\left\{I_{a}^{\alpha}\left(|w(b)||g(b)|^{q}\right)\right\}^{\frac{1}{q}} .
$$


Proof. Let $\varphi:=\frac{1}{p}+\frac{1}{q}<1, \zeta_{1}=\varphi p<p$ and $\zeta_{2}=\varphi q<q$. Then $\zeta_{1}>1, \zeta_{2}>1$ with $\sum_{i=1}^{2} \frac{1}{\zeta_{i}}=1$.

Hence, from Corollary 1 and Corollary 9, we get (40).

Corollary 12 Let $w, f, g \in C_{l d}\left([a, b]_{\mathbb{T}}, \mathbb{R}\right)$ with $J_{a}^{\alpha}|w(b)|=1$. If $p>1, q>1$ with $\frac{1}{p}+\frac{1}{q}<1$, then for $\alpha \geq 1$

$$
J_{a}^{\alpha}(|w(b)||f(b) g(b)|) \leq\left\{J_{a}^{\alpha}\left(|w(b)||f(b)|^{p}\right)\right\}^{\frac{1}{p}}\left\{J_{a}^{\alpha}\left(|w(b)||g(b)|^{q}\right)\right\}^{\frac{1}{q}} .
$$

Proof. Similar to the proof of Corollary 11.

Remark 9 If we set $\mathbb{T}=\mathbb{Z}$, then we get discrete versions and if we set $\mathbb{T}=\mathbb{R}$, then we get continuous versions of the delta and nabla integral operator inequalities presented in this article.

\section{Conclusion}

Fractional calculus on time scales has attracted the attention of many researchers and has been successfully applied in various fields. In the past several years, by using the time scale Riemann-Liouville type fractional integrals, the fractional integral inequalities and applications have been addressed extensively by several researchers. In this research article, we have presented dynamic inequalities in unified form by using the delta and nabla integrals. Our work shows that many classical inequalities such as Radon's inequality, Bergström's inequality, Rogers-Hölder's inequality, Cauchy-Schwarz's inequality, the weighted power mean inequality and Schlömilch's inequality can be refined and generalized on time scales.

\section{References}

[1] J. Aczél, E. F. Beckenbach, On Hölder's inequality, in "General inequalities 2" (Proc. Second Internat. Conf. Oberwolfach, 1978), 145-150, Birkhäuser, Basel/Stuttgart, 1980.

[2] R. P. Agarwal, D. O'Regan, S. H. Saker, Dynamic inequalities on time scales, Springer International Publishing, Cham, Switzerland, 2014.

[3] G. A. Anastassiou, Principles of delta fractional calculus on time scales and inequalities, Mathematical and Computer Modelling, vol. 52, no. 3-4, 2010, 556-566.

[4] G. A. Anastassiou, Foundations of nabla fractional calculus on time scales and inequalities, Computers \& Mathematics with Applications, vol. 59, no. 12, 2010, 3750-3762. 
[5] G. A. Anastassiou, Integral operator inequalities on time scales, International Journal of Difference Equations, vol. 7, no. 2, 2012, 111-137.

[6] D. Anderson, J. Bullock, L. Erbe, A. Peterson, H. Tran, Nabla dynamic equations on time scales, Pan-American Mathematical Journal, vol. 13, no. 1, 2003, $1-48$.

[7] E. F. Beckenbach, R. Bellman, Inequalities, Springer, Berlin, Göttingen and Heidelberg, 1961.

[8] R. Bellman, Notes on matrix theory-IV (An inequality due to Bergström), Amer. Math. Monthly, vol. 62, 1955, 172-173.

[9] H. Bergström, A triangle inequality for matrices, Den Elfte Skandinaviske Matematikerkongress, 1949, Trondheim, Johan Grundt Tanums Forlag, Oslo, 1952, 264-267.

[10] M. Bohner, A. Peterson, Dynamic equations on time scales, Birkhäuser Boston, Inc., Boston, MA, 2001.

[11] M. Bohner, A. Peterson, Advances in dynamic equations on time scales, Birkhäuser Boston, Boston, MA, 2003.

[12] M. Bohner, H. Luo, Singular second-order multipoint dynamic boundary value problems with mixed derivatives, Advances in Difference Equations, 2006, 1-15.

[13] Z. Dahmani, About some integral inequalities using Riemann-Liouville integrals, General Mathematics, vol. 20, no. 4, 2012, 63-69.

[14] C. Dinu, Convex functions on time scales, Annals of the University of Craiova, Math. Comp. Sci. Ser., vol. 35, 2008, 87-96.

[15] D. M. Bătineţu-Giurgiu, O. T. Pop, A generalization of Radon's inequality, Creative Math. \& Inf., vol. 19, no. 2, 2010, 116-121.

[16] G. H. Hardy, J. E. Littlewood, G. Pölya, Inequalities, 2nd Ed., Cambridge, University Press, 1952.

[17] S. Hilger, Ein Maßkettenkalkül mit Anwendung auf Zentrumsmannigfaltigkeiten, Ph.D. thesis, Universität Würzburg, 1988.

[18] C. H. Hong, C. C. Yeh, Rogers-Hölder's inequality on time scales, International Journal of Pure and Applied Mathematics, vol. 29, no. 3, 2006, 289-309.

[19] D. S. Mitrinović, Analytic inequalities, Springer-Verlag, Berlin, 1970.

[20] J. Radon, Theorie und Anwendungen der absolut additiven Mengenfunktionen, Sitzungsber. Acad. Wissen. Wien, vol. 122, 1295-1438, 1913. 
[21] M. J. S. Sahir, Dynamic inequalities for convex functions harmonized on time scales, Journal of Applied Mathematics and Physics, vol. 5, 2017, 2360-2370.

[22] M. J. S. Sahir, Fractional dynamic inequalities harmonized on time scales, Cogent Mathematics \& Statistics, vol. 5, 2018, 1-7.

\section{Muhammad Jibril Shahab Sahir}

University of Sargodha, Sub-Campus Bhakkar, Pakistan

Faculty of Mathematics

Department of Mathematics

GHSS, 67/ML, Bhakkar, Pakistan

e-mail: jibrielshahab@gmail.com 